\title{
Endogenic Cytotoxic Factors and Formation of the Clinic Forms of Myasthenia
}

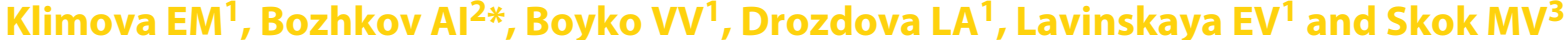

${ }^{1}$ State Institution, V.T. Zaitsev Institute of General and Emergency Surgery of National Academy of Medical Sciences of Ukraine, 61018, Kharkov, Ukraine

${ }^{2}$ Research Institute of Biology, V.N. Karazin Kharkov National University, 61022 Kharkov, Ukraine

${ }^{3}$ Palladin Institute of Biochemistry of the National academy of Sciences of Ukraine, 01601 Kyiv, Ukraine

Corresponding author: Bozhkov Al, Research Institute of Biology, V.N. Karazin Kharkov National University, Ukraine, Tel: +380577075340; Email: bozhkov@univer.kharkov.ua

Received: Jul 01, 2016; Accepted: Aug 08, 2016; Published: Aug 11, 2016

Citation: Klimova EM, Bozhkov Al, Boyko VV, et al. Endogenic Cytotoxic Factors and Formation of the Clinic Forms of Myasthenia. Transl Biomed. 2016, 7:3.

\section{Abstract}

Background: Recently, increased incidence of autoimmune disorders, one of which is myasthenia gravis. The myasthenia is revealed at 3-30 patients out of 100 000 in a year. As it is considered by the most of specialists the central chain in myasthenia development is the formation of antibodies to the nicotinic acetylcholine receptors ( $\mathrm{nAChR}$ ), leading to the blockage of muscle fiber contraction and to the muscle dystrophy. Along with this, there is an assumption of the role of cytotoxic factors in the development of myasthenia gravis. The aim of the present article is to proof working hypothesis about specific role of cytotoxic factors in the formation of various clinical variants of myasthenia gravis.

Method: Patients were divided according to the clinical phenotype of myasthenia gravis: myasthenia without thymus lesion (M), myasthenia with thymus hyperplasia (MH) and myasthenia with thymoma (MT). We used methods of light microscopy to determine the serum cytotoxicity using a cell bioindicator Dunaliella viridis. The determination of antibodies to nicotinic acetylcholine receptors (nAChR), thyroperoxidase (TPO) and antibodies to lungs, liver tissues and elastin was performed by ELISA. And fluorescence microscopy used for determination of expression of clusters differentiation of $\mathrm{CD} 3+, \mathrm{CD} 4+$, CD8+, CD16+, CD19+, CD45+.

Results: The patients with different clinical phenotypes of myasthenia: myasthenia without thymus lesions (M), myasthenia with thymus hyperplasia (MH), and myasthenia with thymoma (MT), have been investigated. It was discovered that the amount of autoantibodies to the $\alpha 1$ and $\alpha 7$ acetylcholine receptor was different among patients with different clinical types of myasthenia. Drastic differences between forms of myasthenia were also identified using a blood serum thyroperoxidase activity. Additionally, we found that all clinical forms of myasthenia could be characterized by the differences in the amounts of IL4 and IL8, compositions of lymphocyte subpopulations, as well as patterns of autoantibodies to the lung tissue, liver, heart, and DNA. Test culture $D$. viridis responded differently to the cytotoxic components of the blood serum in different clinical types of myasthenia. Our results suggest that endogenous factors play a primary role in forming of clinical types of myasthenia. Conclusions: 1) development of myasthenia gravis occurs on the background of cytotoxic factors formed in the organism; 2) endogenous cytotoxic factors have nonspecific effects on the various functional systems of the organism, even the plant objects; 3 ) the organism of patients with myasthenia gravis produce various cytotoxic factors, which influence on different systems of the organism and can lead to the formation of a variety of clinical phenotypes.

Keywords: Autoimmune diseases; Phenotypes; Myasthenia gravis; Cytotoxic factors; Bioindication

\section{Introduction}

From $5 \%$ to $8 \%$ of people suffer from autoimmune pathologies. Untill now there are nearly 80 clinic variants of autoimmune pathologies. The number of autoimmune pathologies increases steadily and the reasons for such increase are not clear [1,2].

The most of autoimmune pathologies is characterized by:

1. High heterogeneity of clinical features [3]; 2. Pathologies arise slowly and lead to adaptive changes of many functional systems and to formation of chronic forms of myasthenia, and it is rather difficult to acquire the remission [4,5]; 3. Most commonly the autoimmune pathologies have the systemic nature; 4 . There could be a lot of trigger factors, and their combination increases the degree of autoimmune pathologies heterogeneity and complicates their diagnosis [6,7]. All these features are indicative for such autoimmune neuromuscular pathology as myasthenia. 
The myasthenia is revealed at 3-30 patients out of 100000 in a year [1]. This disease is manifested at the age of 20-40 and/or 60-70 years and often at women of reproductive age [8]. Reasons of age-related occurrence of myasthenia have no explanation till now $[9,10]$.

As it is considered by the most of specialists the central chain in myasthenia development is the formation of antibodies to the nicotinic acetylcholine receptors (nAChR) [11-13], leading to the blockage of muscle fiber contraction and to the muscle dystrophy $[14,15]$. Often the myasthenia is accompanied by some structure-functional changes of thymus (hyperplasia and thymoma) [16-18]. There is pathology of thyroid and parathyroid glands connected with formation of autoantibodies to thyroperoxidase TPO in such patients $[19,20]$. Consequently, the myasthenia processes in combination with the derangements in a lot of functional systems and so is realized in expressed clinic heterogeneity $[21,22]$.

Marked clinical heterogeneity of the disease is accompanied by a high incidence of violations of vital functions [23]. There are many various embodiments of myasthenia classification, which is based primarily on clinical signs.

1. By nature of myasthenic process: myasthenic episodes, myasthenic state, progressive myasthenia, myasthenia gravis.

2. According to the degree of generalization of movement disorders distinguished: local and generalized forms. Among the local forms of myasthenia are separated: ocular, pharyngeal, facial, musculoskeletal forms. To generalized forms of $\mathrm{MH}$ they attribute myasthenia gravis without myasthenic crises, myasthenia gravis with myasthenic crisis.

3. According to the severity of movement disorders graded as mild, moderate, severe.

4. According to the degree of motor functions recovery in response to anticholinesterase drugs: full recovery, partial recovery, no recovery.

5. In relation to the thymus: derived from the thymus (hyperplasia normoplasia, thymus atrophy); derived from thymoma (epithelial, lymphoid, and lymphoepithelial thymoma).

The main and commonly accepted approaches in myasthenia therapy are the usage of the anti-acetylcholineesterase drugs, non-specific immunosupressors, plasmapheresis and thymectomy [14,24,25]. Unfortunately, these approaches are used without regarding clinic types of myasthenia, and following remission is transient and patients state declines again [26].

One may assume that such high clinical and phenotypical heterogeneity is due to such factors as: 1) genetic polymorphism [27]; 2) diversity of trigger factors and their combination $[28,29]$; 3) formation of cyclic self-maintained processes i.e. manifestation of metabolic memory [22].

As a consequence, at the certain level of hyperactivation of autoimmune processes a wide range of cytotoxic factors is produced with the formation of specific immune-biochemical patterns and one or another clinic form can be manifested depending on their particularities.

Such view at myasthenia allows hypothesizing the role of cytotoxic components forming due to the hyperactivity of immune system and resulting in the induction of catabolism in tissues.

The core of the network myasthenia model is that the content of cytotoxic factors (catabolism products) increases in avalanche-like way according to the proteasome system activation and changes of immune responses to the different triggers factors. In its turn they participate in the formation of specific myasthenic immunological, hormonal and biochemical patterns. The wide range of variants of such metabolic patterns may be generated due to the cytotoxic factors $[30,31]$.

For the classification the cytotoxic factors can be combined in groups.

The aim of the present article is the proof of this working hypothesis. The patients with myasthenia were divided by three clinic phenotypes: myasthenia without thymus lesion (M), myasthenia with thymus hyperplasia (MH) and myasthenia with thymoma (MT).

We determined the presence of cytotoxic factors by cell immobilization reactions, changes in cell shape and formation of cell aggregates in a test culture of Dunaliella viridis, and also relation of cytotoxicity to the content of the autoantibodies to various components of tissue, proinflammatory interleukins in some lymphocyte subpopulations.

\section{Materials and Methods}

\section{Materials of investigation}

495 patients with myasthenia and morphological and functional changes in the thymus - hyperplasia and thymoma have been observed. The first group consisted of 134 patients with myasthenia without morphological and functional changes in the thymus (M); the second group consisted of patients with myasthenia with thymus hyperplasia (MH) - 244 patients; the third group - 117 patients with myasthenia and thymoma (MT) (Table 1).

The study involved 495 patients with myasthenia of varying severity and localization of process (synaptic disorder of skeletal bulbar muscle, chewing and breathing muscles), with disease duration from 6 months to 2 years. In the treatment of myasthenia was used standard protocol, comprising: anticholinesterase therapy, steroid hormones, cytostatics, plasmapheresis, thymectomy and thymomectomy. Efficacy of treatment, which was judged by the duration of remission, was from $35 \%$ to $76 \%$, in different groups of patients. Surgical treatment was the most effective treatment among patients with lymphoepithelial thymoma. The death of $3 \%$ of patients 
was observed due to myasthenic and cholinergic crisis in the early postoperative period.

Table 1 Distribution of patients by gender, age and clinical phenotypes of myasthenia.

\begin{tabular}{|l|l|l|l|}
\hline $\begin{array}{l}\text { Group of } \\
\text { patients }\end{array}$ & \multicolumn{3}{|c|}{ The clinical phenotype of myasthenia } \\
\hline & $\begin{array}{l}\text { M (Myasthenia } \\
\text { without thymus } \\
\text { lesion) }\end{array}$ & $\begin{array}{l}\text { MH (Myasthenia } \\
\text { with thymus } \\
\text { hyperplasia) }\end{array}$ & $\begin{array}{l}\text { MT } \\
\text { (Myasthenia } \\
\text { with thymoma) }\end{array}$ \\
\hline $\begin{array}{l}\text { Number of male } \\
\text { patients }\end{array}$ & 114 & 65 & 76 \\
\hline Age, years & $32-57$ & $17-45$ & $45-70$ \\
\hline $\begin{array}{l}\text { Number of } \\
\text { female patients }\end{array}$ & 62 & 145 & 33 \\
\hline Age, years & $27-35$ & $24-57$ & $56-69$ \\
\hline
\end{tabular}

In the group of patients with $M$ generalized forms of myasthenia with mild, moderate and severe violations of bulbar, pharyngeal and respiratory muscles were observed. In the groups of patients with $\mathrm{MH}$ and MT was observed muscle damage zones with electromyography confirmation. In groups with different types of morphological and functional changes in thymus there were patients with varying degrees of severity of the neuro-transmitter lesions. Progressive muscular dystrophy was observed in $24 \%$ of patients with $\mathrm{MH}$. In patients with MT were observed mainly mild myasthenic disorders, but in the postoperative period they were observed myasthenic and cholinergic crises.

Patients with generalized myasthenia obtained anticholinesterase drugs in an amount corresponding to the severity of the neurotransmitter disorders - kalimin of 1 to 5 tablets per day, some patients received the drug neostigmine methylsulfate and steroid hormones (drugs were selected individually, gradually reducing the dose of $100 \mathrm{mg}$ to $5 \mathrm{mg}$ ).

With the development of myasthenic crises among patients receiving mechanical ventilation in the intensive care unit, steroids administered intravenously from 50 to $100 \mathrm{mg}$, according to individual indications. Throughout observations including postoperative period, the cholinesterase activity was monitored in serum of patients for selection anticholinesterase drugs to prevent cholinergic crisis.

\section{Determination of cell motility, cell shape and formation of cell aggregates with test cells $D$. viridis}

Investigation of the cytotoxicity of the serum was performed using the cell-test based on $D$. viridis Teodor. The synchronized culture of unicellular algae was applied; the characteristic feature of these algae is the lack of a cell wall. In immunoassay plate was added serum and test system in equal volumes. After incubation for $\mathbf{3 0}$ minutes by microscopy the cytotoxic effect of sample components on the cells of $D$. viridis in test system was evaluated. We registered such cell responses: morphological (cell shape) and functional changes (loss of motility, loss of flagella, aggregate formation). After incubation, the cell suspension of investigated serum with $D$. viridis we calculated the number of abnormal cells: rounded, motionless and aggregated cells and calculated their percentage in comparison with the control [32].

For the control test the ratio of the spontaneous cytotoxicity (CSP) was calculated by the formula (I):

$$
C_{S P}=\frac{{ }^{M_{C}+F_{C}+A_{C}}}{3}
$$

Where, the MC - the percentage of cells with a modified form of the control; FC - the percentage of cells with altered functional properties in control; AC - percentage of aggregated cells in the control.

In formula (II) induced cytotoxicity coefficient calculated for each serum obtained from patients with various pathological conditions:

$$
C_{I}=\left(\frac{M_{I}+F_{I}+A_{I}}{3}-C_{S P}\right) \cdot \frac{1}{C_{S P}} \ldots \ldots \ldots . .(\text { III })
$$

Where, $\mathrm{MI}$ - the percentage of cells with a modified form after incubation with certain pathologic serum; FI - the percentage of cells with altered functional properties after incubation with certain pathologic serum; Al - percentage of aggregated cells after incubation with certain pathologic serum; CSP - the coefficient of spontaneous cytotoxicity control.

\section{The determination of antibodies to nicotinic acetylcholine receptors ( $\mathrm{A} A \mathrm{Ch} R$ )}

To determine the presence of antibodies to the $\alpha 1$-subunit muscular nAChR $\alpha 7$-subunit neuronal nAChR, the test system of immunoassay on the solid support was used. The resulting complex "antigen-antibody" was detected by a conjugate, which catalyzes the cleavage of peroxidase substrate (hydrogen peroxide), causing a color change of indicator. Optical density measurements were performed at $450 \mathrm{~nm}$ on ELISA analyzer Stat Fax 3200 (USA) [33].

\section{The determination of an
thyroperoxidase (TPO) in serum}

TPO antibodies in the serum were determined using immunoassay on solid support. Measurement of the optical density of the complex "antibody-antigen" was performed at $450 \mathrm{~nm}$ on ELISA analyzer Stat Fax 3200 (USA) [20].

\section{Method of determination of the concentration of antibodies to lungs, liver tissues and elastin}

Organ-specific antibodies (OSA) to the tissues of the lungs, heart and liver, anti-elastin were determined using enzyme immunoassay system ("Navina", Russia). The principle of the method is the interaction of tissue specific antigen, adsorbed on the plate, with the antibodies to them, contained in serum. The resulting complex "antigen-antibody" is detected by a 
conjugate, which catalyzes the cleavage of peroxidase substrate (hydrogen peroxide), causing a color change of indicator. The intensity of color is in direct ratio to the amount of antibodies to antigens investigated. The intensity of the components in the patient's serum binding with specific antigens was calculated relative to control serum binding reaction taking the level of optical density of control serum responses to each of the antigens as $100 \%$ [34].

Expression of clusters of differentiation of CD3+, CD4+, CD8+, CD16+, CD19+, CD45+ was assessed by indirect immunofluorescence using monoclonal antibodies (LRS "Sorbent", Russia) labeled with FITC - fluorescent dye. When this method is setting up the specific monoclonal antibodies labeled with FITC (not directly but through the secondary serum) associate with a corresponding cell surface antigen. The cells stained by corresponding antibodies were visualized by fluorescent microscope. Character induction of expression associated with the presence of cytotoxic factors serum [35].

\section{Determination of the interleukins concentrations in serum}

To measure the level of IL-4,8 the solid-phase immunoenzyme method with the horseradish peroxidase as an indicator enzyme is used. The activity of bound peroxidase was measured with an automatic microplate photometer at a 450 $\mathrm{nm}$ wavelength [20].

\section{Statistical analysis}

The results obtained were processed statistically using the Wilcoxon-Mann-Whitney criterion and Student's t-test, using the software package "Statistika V.6".

\section{Results}

The reaction of the cell bioindicator to cytotoxic factors of blood serum of patients with different clinical phenotypes of myasthenia

Since the composition and nature of complex of cytotoxic factors are unknown, and it is not yet possible to determine them clinically by analytical methods, the testing of this hypothesis was based on the use of non-specific cell bioindicator $-D$. viridis.

Unicellular microalgae $D$. viridis is characterized by: 1 - the absence of a cell wall, thus allowing. the interaction and/or penetration of low molecular cytotoxic factors in the cell; 2 high sensitivity to the presence of cytotoxic factors, regardless of their nature; 3 - variety of responses to stress effects (loss of mobility, changes in cell shape, secretion exometabolites, the formation of cell aggregates with different characteristics); 4 $D$. viridis responses to stressors are interrelated and characterized by the hierarchy, which may allow the identification of cytotoxic factors depending on the response of the bioindicator [32].

Thus, in control cell culture $90-95 \%$ of cells are mobile and only $5-10 \%$ of the cells are motionless. Loss of motility of $D$. viridis cells is associated with both a reaction to stress exposure, which are accompanied by inhibition of their metabolic activity, and with the natural process of the formation of cysts in sexual reproduction. Under optimal conditions, $D$. viridis reproduces vegetatively and do not lose mobility.

Determination of the amount of motile cells in the control culture after 30 minutes, 90 minutes and 2 hours after application of $50 \mu \mathrm{l}$ of saline had no effect on cell motility (Figure 1). The same amount of healthy donors' serum also had no effect on cell motility.

In the case if $50 \mu \mathrm{l}$ of the blood serum of patients with myasthenia without thymus lesion (M) was added to the testculture $55 \%$ of cells, lose their motility in 30 minutes. After 2 hours, only $5 \%$ of cells in culture were mobile. In the case of patients with thymus hyperplasia ( $\mathrm{MH}$ ) in 30 minutes, $95 \%$ of cells lose their mobility (Figure 1 ).

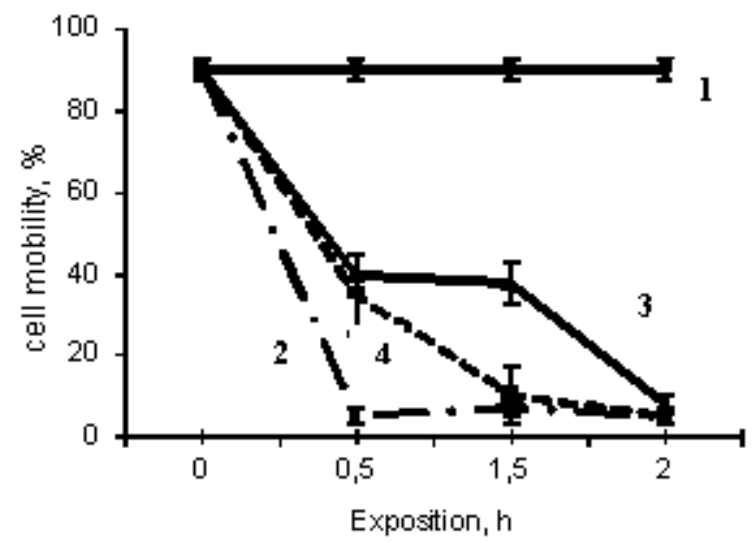

Figure 1 Cell motility of test-culture at different exposures after making into culture of blood serum of healthy donors (1), of patients with myasthenia (2), of patients myasthenia with thymus hyperplasia of the (3) and of patients with myasthenia gravis with thymoma (4).

Another character of cell immobilization was observed after addition to the test-culture of $50 \mu$ of blood serum of patients with myasthenia with thymoma (MT). After 30 minutes and 90 minutes of incubation only $40 \%$ of cells retain mobility, however by 2 hours of incubation in the presence of serum components only $8 \%$ of cells were mobile (Figure 1).

These results suggested that: 1 ) in serum of all the patients with myasthenia there are cytotoxic factors inhibiting the mobility of bioindicator; 2) the effect of inhibition of form change and cell mobility and temporal characteristics of its development were different in the cases of different clinical types of myasthenia. One may assume that the composition and/or the quantity of cytotoxic factors at the different clinical phenotypes can be different.

If the cytotoxic components in the blood serum of patients with myasthenia and hyperplasia immobilize cells during 30 minutes and further this characteristics didn't change, in the 
case of myasthenia it increased by 3.6 times from 30 to 90 minutes and after the immobilization rate decreased, and in the case of MT, on the contrary, the sharp immobilization was observed from 0 to 30 minutes and from 90 minutes to 2 hours it not changed additionally.

Along with the immobilization of $D$. viridis cells, the cytotoxic components of blood serum of patients with myasthenia influenced the cell shape changes of $D$. viridis.

In the control culture of $D$. viridis cell were pear-shaped (near $42 \%$ ), ellipsoid (52\%) and roundish, immobile (7\%) cells (Figure 2).
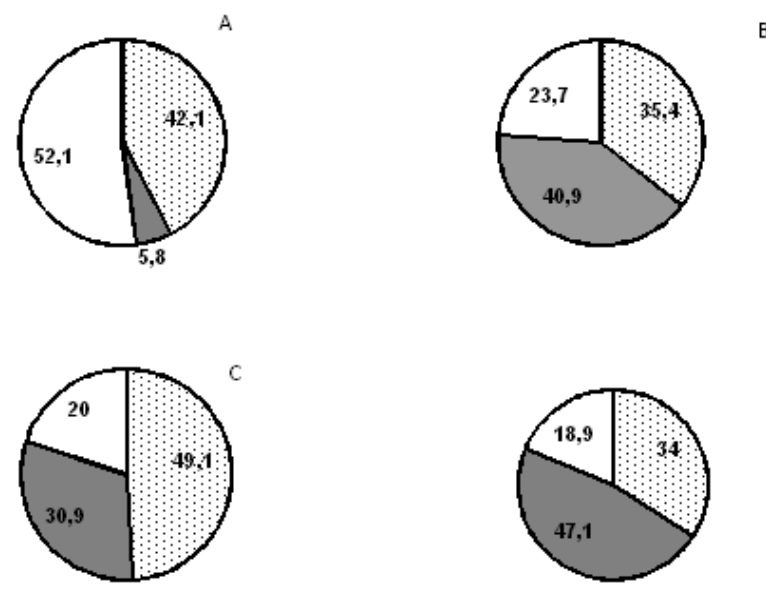

Figure 2 Ratio of cell forms of $D$. viridis 2 hours after introduction of $50 \mathrm{ml}$ of serum from healthy donors $(A)$, serum from myasthenia gravis patients without thymus lesions patients (B), serum from myasthenia with hyperplasia of the thymus patients (C) and serum from myasthenia with thymoma patients (D) into culture medium

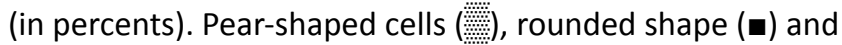
ellipsoidal shape (口).

If the patients' serum was added to the test-culture, the pattern of cell shapes changed. In myasthenia without thymus lesion, the number of round cells corresponded to $25 \%$, while $\mathrm{MH}$ (myasthenia with thymus hyperplasia) revealed on the background of the maximum number of cells that changed under the influence of the cytoskeleton cytotoxic factors, which corresponded to $43 \%$. A myasthenic factors of blood serum of patients with myasthenia gravis in the background thymoma changed shape of $D$. viridis cells from circular to elliptical in $35 \%$ of cases.

First of all the amount of roundish cells increased and the amount of normal ellipsoid ones decreased, but the amount of pear-shaped didn't change (Figure 2). These patterns didn't differ significantly between clinical phenotypes of myasthenia. The ratio of ellipsoid/roundish cells changed most demonstrably. If in control this ratio was 9.0, in the myasthenia patient it was $0.40,0.58$ and 0.65 correspondingly for $\mathrm{M}, \mathrm{MH}$, MT.
The most significant changes in $D$. viridis cells the testculture were related to emergence cell aggregates induced by components of serum of patients. These aggregates were different for various clinical forms of myasthenia (Figure 3 ).
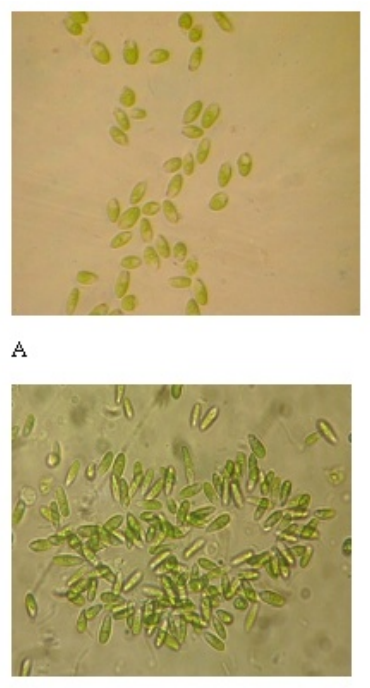

$\mathrm{C}$
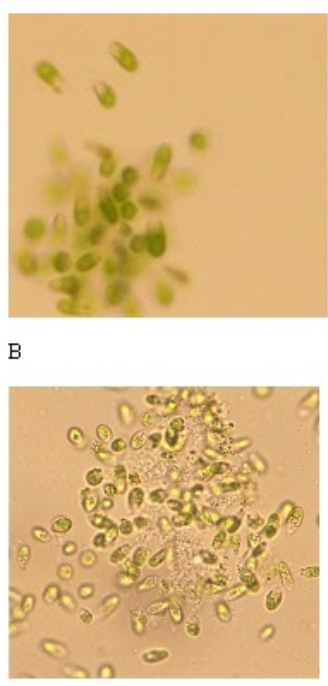

Figure 3 The formation of cell aggregates addition the culture of $D$. viridis in serum of patients with myasthenia without thymus lesions (B), myasthenia with thymus hyperplasia of the (C), myasthenia with thymoma (D). Control culture (A) (objective $\times 40$ ).

In the case of patients with $M$, the number of aggregates can reach 60 within of eyeshot, but the amount of cells in aggregates is not more than from 3 to 20 cells (Figure 3 ).

In the case of the patients with $\mathrm{MH}$ amount of cells in aggregates were larger and they have an elongated shape, and at MT the amount of the forming aggregates were larger, they have another shape (Figure $\mathbf{3}$ ).

Consequently, in blood serum of patients with myasthenia there are cytotoxic factors influencing not only the cells of own organism but also the plant cells in culture suggesting the wide range of biological activity or of the presence of specific receptors in the unicellular algae [36]. Taking into account the different responses of bioindicator on the serum components of patients with different forms of myasthenia one may assume that the amount of such components can be different.

In further investigations of the study of bioindicator ( $D$. viridis) answer to patient's serum it was carried out studies in dynamics of treatment. First study was carried out before operation of thymectomy, the second study on the third day after surgery, third study - on the fifth day after surgery.

Postoperative period at patient V. was complicated by myasthenic crisis and at the 6th day the patient died (Figure 4A).

Serum components of patient $\mathrm{V}$. at the first study resulted in an increase in number of rounded (in 6 times) and motionless (in 7 times) cells. In the early postoperative period after 
thymectomy the number of modified cells $D$. viridis falls almost 2-fold (Figure 4A). And on the 5th day after the operation myasthenic crisis was observed and serum of patients $V$. has a stronger negative impact on cell biological indicator, which was manifested in the increase in the number of round and fixed cells by an average 2-fold compared with the second investigation. The appearance of the cell aggregates was observed.
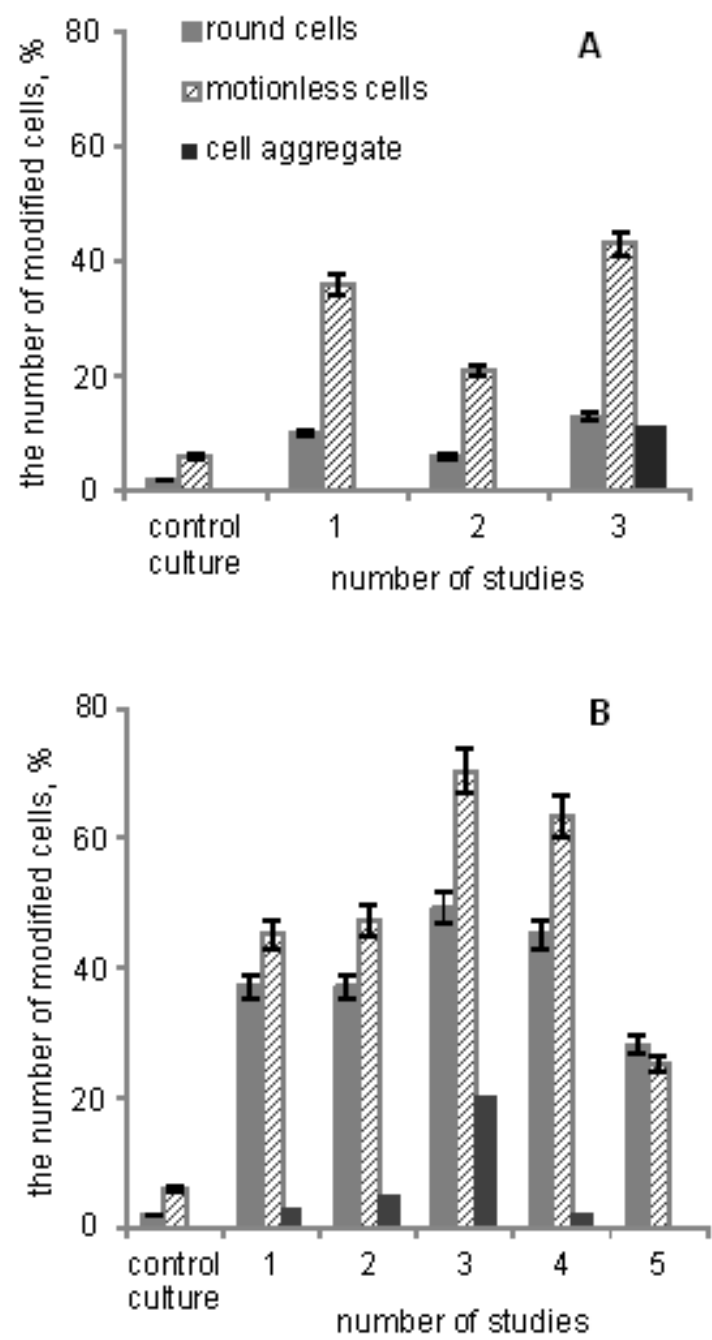

Figure 4 Change in response of bioindicator $D$. viridis to a set of myasthenic cytotoxic factors in patient $\mathrm{V}$. with unfavorable outcome (A) and in patient $\mathrm{S}$. with favorable outcome (B).

Myasthenia of patient S. proceeded with periods of remission and complications and the positive outcome of the disease (Figure 4B). Investigations were carried out in dynamics: first study - at the time of admission of the patient to the clinic, second study - after five days, third study - on the 7 th day from the date of receipt, forth study - on the 10th day, fifth study - on the 25 th day from receipt.

Figure 4B shows the dynamics of changes in reaction bioindicator $D$. viridis on cytotoxic components of blood serum of the patient S., who was not operated and received conservative treatment, including anticholinesterase therapy, immunomodulators, methods of gravitational surgery plasmapheresis and intravenous injection of exogenous human IgG.

The first study corresponds to a state of remission, which was the result of a multi-component therapy. The number of rounded cells and still higher than the reference value in the average 6 -fold. At the time of the second study within five days after receipt, we observed a slight increase in the number of aggregated cells bioindicator. On the 7th day (3rd research) during the exacerbation of myasthenia gravis have seen a significant change in the biological indicator $D$. viridis (increasing the number of round cells and increase the number to $70 \%$ of the cells that have lost their mobility). The number of aggregated cells in this period increased by $15 \%$. Sessions plasmapheresis caused positive changes in the general condition of the patient S., as well as reducing the number of aggregated cells $D$. viridis to $3 \%$, and a decrease in the number of cells to change the shape and the loss of mobility. In subsequent periods survey completely disappeared and cell aggregates bioindicator significantly decreased the number of rounded cells and stationary (in comparison with the 3rd study) after their incubation with patient serum. Response of bioindicator $D$. viridis to myasthenic cytotoxic factors in these two patients was variable and dynamic fit of the disease.

\section{Contents of autoantibodies in patients with various clinical forms of myasthenia}

There is evidence of involvement in the pathological process of a variety of mechanisms. This primarily relates to the identification of spectrum AAB [37]. The concept of the pathogenesis of myasthenia gravis, including heterogeneity and differing vectors of $A A B$ and the difference in their interaction with cholinergic receptors and other targets will determine the criteria for the diagnosis and treatment of diseases of individual paths [38].

Contents of autoantibodies (AAB) to nicotinic $\alpha 1$ subunits determinants of acetylcholine receptors in patients with myasthenia increased by 1.6-2.2 times depending on the clinical forms of pathology.

Among patients with myasthenia without thymus pathology the content $A A B$ to $\alpha 1$ subunits of acetylcholine receptors was 1.7 times higher compared with the reference values; in the case of myasthenia with thymus hyperplasia it was 2.3 times higher, and in the case of myasthenia with thymoma it was 1.6 times more (Figure 5A).

The content of antibodies to AChR $\alpha 7$ subunits in group $M$ was increased 1.9-fold. In the group of patients with $\mathrm{MH}$ and MT AAB to $\alpha 7$ subunits AChRs was increased by 1.3 and 1.25 times, respectively, compared with the reference value (Figure 5B).

Consequently, the spectrum of autoantibodies to various determinants of the nAChRs was different among patients with different clinical phenotypes of myasthenia and their 
number was also different in these clinical phenotypes of myasthenia.

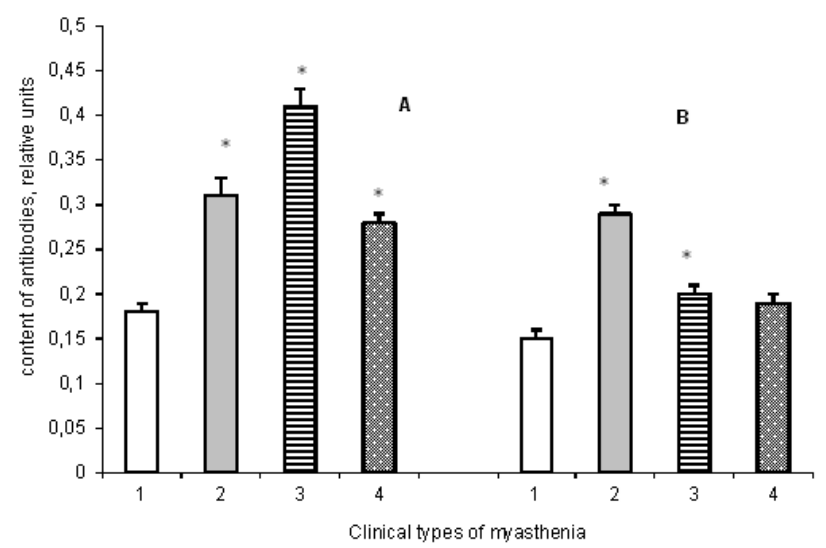

Figure 5 Content of autoantibodies to $\alpha 1$ subunits of the acetylcholine receptors and to $\alpha 7$ subunits of the acetylcholine receptors in serum blood $(B)$ in a reference group (1), patients with $\mathrm{M}(2)$, with $\mathrm{MH}(3)$ and $\mathrm{MT}$ (4).

One of the pathogenetic links of myasthenia gravis may be disorders in enzyme activity due to the presence of specific AAB.

The content of AAB to TPO - an enzyme that is involved in the conversion of thyroglobulin in thyroid hormones thyroxine and triiodothyronine were studied.

Content of autoantibodies to thyroid peroxidase (TPO) increased by 15 times as compared with the control in the case of myasthenia without thymus pathology. While the content of autoantibodies to TPO was even less than the control by 2 times in the case of $\mathrm{MH}$ and 2.4 times compared to control in patients with myasthenia with thymoma (Figure 6).

Consequently, such a change in different directions of antibodies to TPO in patients with different clinical forms of myasthenia indicates the presence of different patterns of autoantibody. Obviously, in these patients, against the background of combined lesions of the thymus and thyroid gland at periglandular syndrome different metabolic patterns are formed.

It could be expected that the number of autoantibodies to the components of various tissues in patients with myasthenia will be increased.

The content of $A A B$ to the components of cell membranes of the lungs in patients with myasthenia was also increased. Thus, in patients with myasthenia without thymus lesion $A A B$ was increased by 2.5 times, with thymic hyperplasia by 7 times, and with thymoma by 5 times in comparison with control (Figure 7A).

Study of serum content $A A B$ to hepatocytes revealed increasing concentrations of the components $A A B$ hepatocyte membrane, which was greatly increased by 25,22 and 25 times among patients with $\mathrm{M}, \mathrm{MH}$ and $\mathrm{MT}$, respectively, compared to the control level (Figure 7B). Such high level of autoantibodies to components of the liver indicates a possible change in its functional activity in these patients.

The content of the $A A B$ to cardiomyocytes also was increased in patients with $\mathrm{MH}$ and $\mathrm{MT}$ (Figure $7 \mathrm{C}$ ).

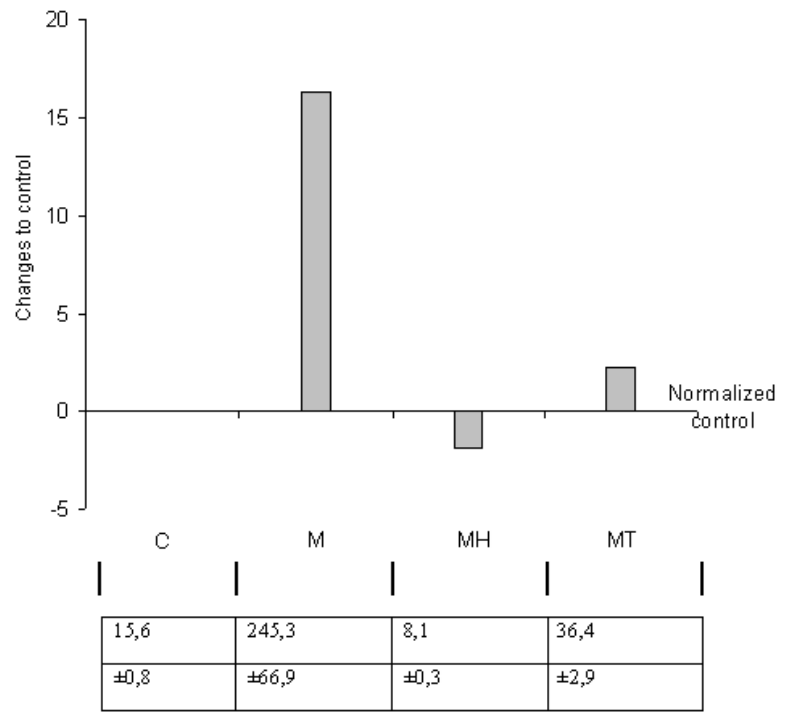

Figure 6 The content of autoantibodies to TPO in a group of healthy volunteers $(K)$, patients with myasthenia without thymus lesion (M), patients with myasthenia with thymoma (MT) and of patients with myasthenia with thymus hyperplasia ( $\mathrm{MH}$ ) (data in the table) and the change in the content of autoantibodies in patients on compared to control (histogram).

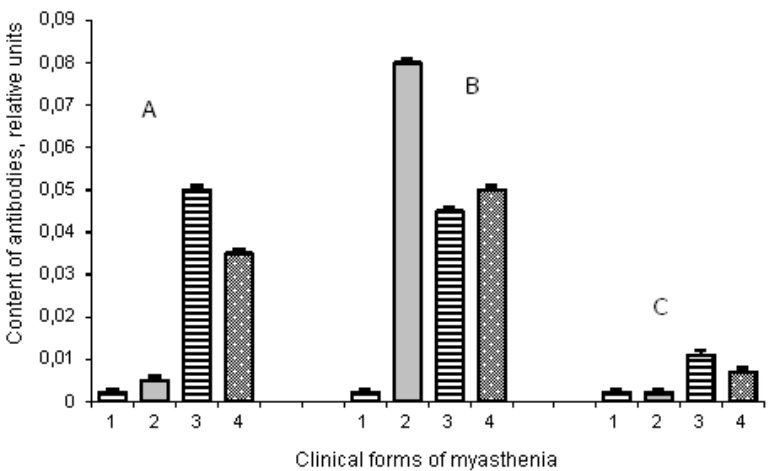

Figure 7 The contents of autoantibodies to components of the tissue (I): lung (A), liver (B), heart (C) in the control group (1), in patients with myasthenia gravis without thymus lesions (2), myasthenia with thymus hyperplasia (3), myasthenia with thymoma (4).

The content of autoantibodies to connective tissue protein elastin in patients with myasthenia also was determined. In patients with myasthenia without thymus lesion and with thymus hyperplasia the autoantibodies to this protein were 
not detected, but their content was increased 10-fold compared with control in patients with myasthenia with thymoma. Therefore, in these patients there may be degradation of the extracellular matrix.

Therefore, in patients with myasthenia increasingly produce antibodies to components of hepatocytes, and this is independent of the phenotype of myasthenia, they also produce antibodies to the cells of the lung and their number depends on the phenotype of myasthenia gravis. In individual patients with $\mathrm{MH}$ and MT we have seen a significant increase of $A A B$ to the cardiomyocytes.

This range of $A A B$ may have marked destructive actions and cause to alteration of tissue that contributes to the emergence of various cytotoxic factors in patients with myasthenia gravis. The presence of autoantibodies having hydrolytic activity causes the formation of cytotoxic products abzymes and nonspecific degradation of macromolecules [39].These data identify patients with different clinical phenotypes myasthenia individual metabolic patterns that differ from the norm.

\section{Characteristics of the lymphocyte subsets}

The lymphocytes are known to regulate the antigenic homeostasis of the body. A variety of antigenic factors and microenvironment induces the expression of clusters differentiation of different lymphocytes subpopulations.

In patients with different clinical phenotypes of myasthenia the ratio of lymphocyte subpopulations: CD3+; CD4+; CD8+; CD16+; CD19+ were determined.

It was found that in the group of donors without autoimmune pathologies, more than $50 \%$ of lymphocytes belong to the $\mathrm{CD} 3+$ subpopulation. In these donors $30 \%$ to $40 \%$ of lymphocytes belong to CD4+ subpopulation, from $20 \%$ to $35 \%$ of lymphocytes were CD8+; CD19+ - $17 \%$ to $20 \%$, and the least of them was CD16+ (8\% to $10 \%)$ (Figure $8 \mathrm{~A})$.

Among the patients with myasthenia without thymus lesion the expression of CD3+ (common T-lymphocytes) and CD8+ (killers/suppressors) was reduced, and the content of other lymphocyte subpopulations did not differ significantly from healthy donors (Figure 8B).

In the case of myasthenia with thymic hyperplasia a significant increase of CD4+ (T-helper cells) and CD16+ (NK) content was observed and $\mathrm{CD} 8+$ content was reduced as compared with the control (Figure $8 \mathrm{C}$ ).

The patients with myasthenia with thymoma were characterized by reduced expression of $\mathrm{CD} 3+, \mathrm{CD} 8+, \mathrm{CD} 16+$ and $\mathrm{CD} 19+$ content was increased in them compared with healthy donors (Figure 8D).

Consequently, the ratio of lymphocyte subpopulations in patients with myasthenia has been changed compared with the control group. Each of the clinical forms of myasthenia has been a specific pattern of lymphocyte subpopulations (Figure 8).

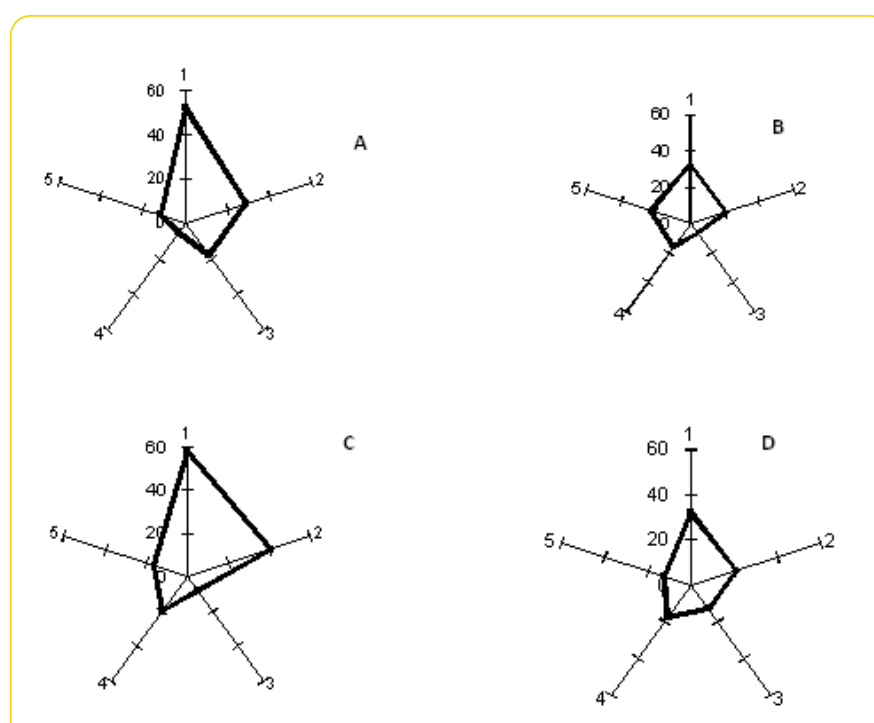

Figure $\mathbf{8}$ The content lymphocyte subpopulation in the control group $(A)$ in patients with myasthenia gravis without thymus lesion (B) with myasthenia with thymus hyperplasia (C) and myasthenia with thymoma (D). Content on axes $\mathrm{CD} 3$ $+(1), \mathrm{CD} 4+(2), \mathrm{CD} 8+(3), \mathrm{CD} 16+(4), \mathrm{CD} 19+(5)$.

The change of lymphocyte subpopulation composition at myasthenia reflects: features and degree of response to antigenic background; the nature of the communication between subpopulations of immune cells, i.e., forming of immunocompetent cells pattern and individual set of cytokines in the body.

It should be noted that these measures, which have been identified in a large number of patients remained quite variable, what reflects the high individual variability of immunoreactivity of the organism.

\section{The content of IL-4 and IL-8 in patients with different clinical phenotypes of myasthenia}

It is known that the role in the system development of autoimmune pathologies play proinflammatory cytokines, acute phase proteins and other biologically active factors which may be produced in different subpopulations of lymphocytes. In this relation the content of the proinflammatory interleukins IL-4 and IL- 8 can be indicative in patients with myasthenia. IL-4 is central in the regulation of the immune response and provides differentiation Th2 lymphocytes. IL-4 is growth factor of B-lymphocyte, inducer of DNA synthesis in lymphocytes and increases the expression of HLA II. IL- 8 is associated with induction of IL-1 $\beta$, enhances intercellular adhesion properties [40]. These cytokines describing features of differentiation of immune cell producers, we used as a criterion for the presence of cytotoxic factors at bioindication.

The content of IL-4 was found to be increased in patients with myasthenia compared with control levels from 3.9 to 9.5 times depending on the clinical form of $\mathrm{M}$. Its maximal content 
was revealed in patients with $\mathrm{MT}(666 \mathrm{mmol} / \mathrm{l})$ at $70 \mu \mathrm{l} / \mathrm{l}$ in the control (Figure 9A).

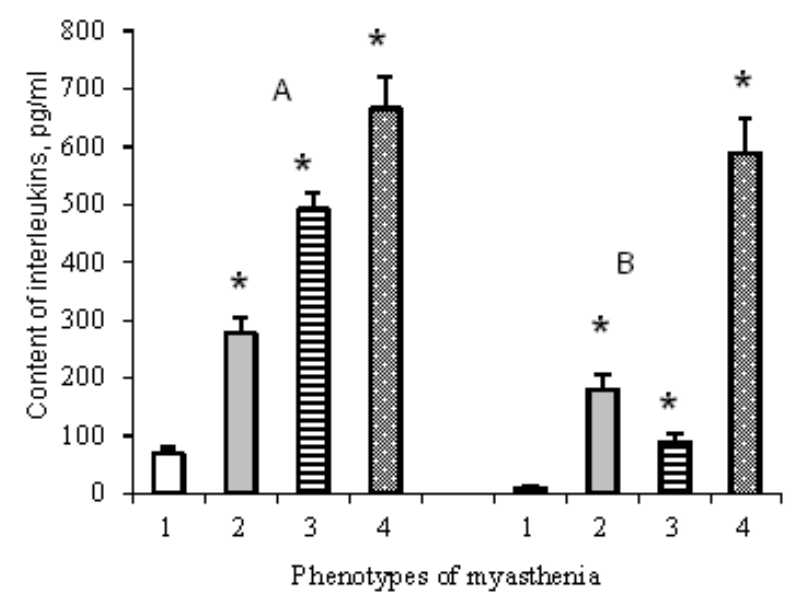

Figure 9 The content of interleukin-4 (A) and interleukin-8 (B) in control group (1), of patients with myasthenia (2), of patients myasthenia with thymus hyperplasia (3) and of patients with myasthenia with thymoma (4)

The content of IL-8 in these patients has been increased to a greater extent than the reference level. Therefore, its content was 18 times higher in patients without thymus lesions (M), 8.8 times in patients with $\mathrm{MH}$ and 59 times in patients with MT versus control (Figure 9B).

Consequently, the development of myasthenia was accompanied by increase of content both IL-4 (in 3.9-9.5 times), and IL-8 interleukins (8.8-59 times) as compared to a control level. The greatest content of IL-4 and IL- 8 was observed at the myasthenia with thymoma and was by 9.5 and 59 times more, respectively (Figure 9).

Such a significant change in the content of the studied interleukin may indicate a profound changes not only the spectrum of cytokines and other cytotoxic factors accompanying inflammation. The development of autoimmune pathologies may be accompanied by the formation of various unidentified cytotoxic factors causing alteration of tissues.

Consequently, in the formation of clinical myasthenia phenotypes can make significant contribution the various biologically active factors that induce different patterns and metabolic memory.

\section{Discussion}

History of description of myasthenia gravis syndrome, research of this syndrome and development of ways to treat it is an interesting and instructive story. This pathology was first described by English physician T. Willis in 1672.
In 1894, J. Jolly defined the electrophysiological characteristics of myasthenia gravis and suggested the name for this disease - myasthenia gravis pseudoparalytic.

C. Weigert in 1901 was the first who described the sarcomatous degeneration of the thymus at the deceased from myasthenia gravis. Involvement of the thymus in the development of myasthenia gravis was proved later, and this fact is the core of the modern theory of the thymogeneous nature of myasthenia [41]. The high frequency of the histological changes observed in the thymus, the presence of large amount of T-lymphocytes in it and serum stimulating production of antibodies to the nicotinic acetylcholine receptor attests in favor of that. The evidence of the important role of the thymus in the development of myasthenia gravis is the fact that many patients after total thymectomy had an experience of clinical improvement [25].

The next important step in the myasthenia study was the work by Mary Walker in 1934, which showed that Curariform factors had an influence on the expression of myasthenia gravis. This work became the basis for wider application of anticholinesterase drugs in the treatment of myasthenia gravis [42].

In the 50-60s of the twentieth century two hypotheses of possible mechanisms of myasthenia gravis were also discussed. The first - a disorder of muscle function due to the appearance of toxic substances in the blood that are absent in norm [43]. The second - the insufficient activity of the neurotransmitter - acetylcholine, which resulted in its destruction by cholinesterase, lack of its synthesis or decreased sensitivity towards acetylcholine [23,44].

The active studying of the role of the immune system in the pathogenesis of myasthenia gravis started in the 80 s of the twentieth century. It was shown that this pathology has an autoimmune nature. In $1993 \mathrm{~K}$. Oda detected antibodies to acetylcholine receptor (AChR) subunits [45]. However, antibodies to AChR were not detected among all patients, but only of $50 \%$ to $60 \%$ [3]

Numerous studies shown that patients with myasthenia gravis had an increased content of antibodies to Titin (striated muscle protein), to the cells of the thymus [46], to ryanodine receptors [47] and others.

This pathology is not always accompanied by the involvement of the thymus. Moreover, immunological and biochemical parameters at patients with myasthenia gravis can vary greatly. This entire are impeded the diagnosis and chose of options for the treatment, and indicates the diversity of the mechanisms of this disease.

Consequently, myasthenia gravis exhibits extremely high heterogeneity of etiology and of pathological factors, and it may have different mechanisms of development.

The results of this work indicate the high heterogeneity of immunological parameters of myasthenia. Therefore, if one considers the variability in the parameters of different clinical phenotypes of myasthenia gravis, one can see absolutely 
different patterns of distribution for different clinical forms of myasthenia gravis.

The high variability of clinical, biochemical and immunological parameters of myasthenia is admitted by many authors $[48,49]$. Such variability is explained by individual, genetic and even geographical factors [50].

Unfortunately, the proper attention to the role of the cytotoxic factors in development of myasthenia was not given proper attention on the background of mentioned above important results. However, hyperactivity of the immune system which can be induced by a variety of factors, and especially infectious agents, is accompanied by the formation of a variety of autoantibodies.

Previously, it was shown that myasthenia gravis is always developed on the background of mix-infections [51].

This does not mean that myasthenia gravis has an infectious nature. We suppose that on the background of "predisposition" mix-infections trigger the autoimmune process, accompanied by the formation of endogenous cytotoxic factors.

It is known that autoantibodies possess proteolytic activity, and protein hydrolysis products may have cytotoxic properties [52]. The increased contents of autoantibodies to components of liver, lungs, and other tissues could result in increased content of products of catabolism in various organs and their nature may be different. It is obvious that not all of them have cytotoxic properties, but that myasthenia is accompanied by the formation of cytotoxic factors evidenced by the results obtained in our experiments using the test culture $D$. viridis.

The results of the influence of (blood) serum components on the culture $D$. viridis, which is a vegetable object and never encounters similar factors in the nature, suggests that in the organism of patients with myasthenia the cytotoxic factors with a not specific activity are formed.

In considering the diversity of $D$. viridis responses: this is the change in cell motility, their form and the formation of cell aggregates, it should be noted that they were different for all the clinical forms of myasthenia gravis.

Moreover, between changes in cell motility, shape and characteristics of the cell aggregates was revealed no connection, i.e., these characteristics varied independently and one may assume that they are induced by different cytotoxic factors. Therefore, among patients with different myasthenia forms there are various cytotoxic factors that may affect the different functions of organism (Figure 10).

In favor of the supposition that the $D$. viridis test reveals the changes induced by cytotoxic factors could be the results of similar responses of $D$. viridis cells after the application of known toxicants [52].

It is important that the loss of cell motility is caused by inhibition of the contractile apparatus of flagella $D$. viridis [53]. Changing the shape of cells $D$. viridis clearly points to the effect of serum components of patients with myasthenia on cytoskeleton and contractile proteins of cells [32].

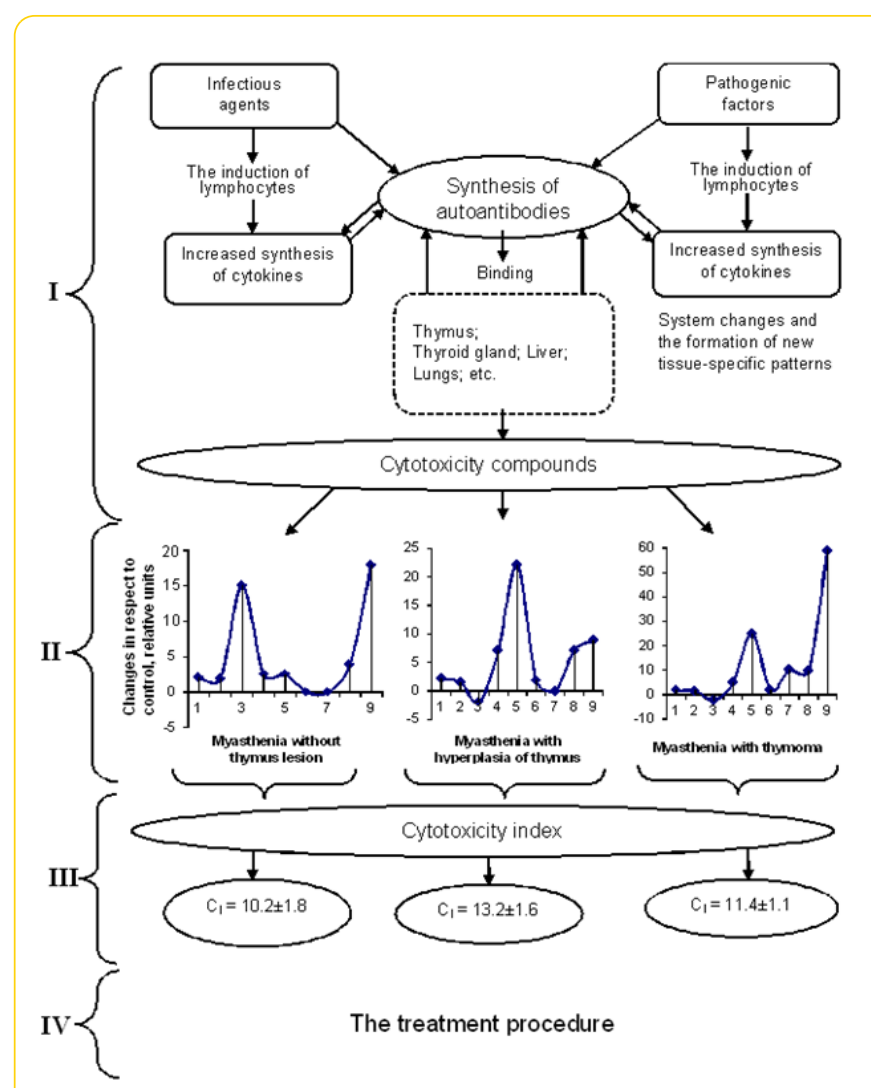

Figure 10 Diagram showing the role of cytotoxic factors in the formation of a variety of clinical forms of myasthenia, cell testing and treatment selection. In the first stage pathological factors and/or infectious agents, induce the synthesis of cytokines and autoantibodies. Autoantibodies are associated with different tissues and further enhance the synthesis of autoantibodies and cytotoxic factors. In the second stage the specific immunobiological patterns for different clinical types depending on the set of cytokines and cytotoxic factors are formed. Induction of the immune response by exogenous and endogenous factors is followed by formation of an extensive network of cytotoxic factors and cytokines that influence the maintenance of a specific immune response and provide the development of the metabolic patterns manifested by different clinical types of myasthenia. On the $y$-axes, the control level is represented as " 0 "; increase in the parameters is in the "+" area; reduction in such parameters is in the "-" area relative to control level. On the $x$-axis, the following parameters are presented: 1. AAB to $\alpha 1-n A C h R ; 2$. AAB to $\alpha 7-n A C h R ; 3$. AAB to TPO; $4 A A B$ to the lungs; 5 . AAB to the liver; 6 . AAB to the heart. 7. $A A B$ to elastin; 8. IL4; 9. IL8. Formed pattern of cytotoxic factors can be tested by bioindicator $D$. viridis (phase III) and selected treatment strategy (stage IV).

The formation of cell aggregates formed by $D$. viridis was described for a long time and is assumed that it is a response of a cell culture on non-specific stress factors [54]. Unfortunately, the mechanisms of this phenomenon are still poorly studied. These results, albeit indirectly but demonstrate that different factors of cytotoxic nature are contained in 
organism of patients with different clinical forms of myasthenia. To assess the presence of complex cytotoxic factors, you can use the integral index of cytotoxicity, which is calculated by the formula II and it was various for the different clinical forms of myasthenia gravis.

Previously we had shown that there is dose dependence between the amount of components from serum of the patients added to $D$. viridis culture and the response of $D$. viridis culture. During the remission the amount of cytotoxic factors in the blood as registered by $D$. viridis response decreased to their absence [55].

\section{Consequently, our results allow concluding:}

Development of myasthenia gravis occurs on the background of cytotoxic factors formed in the organism;

Endogenous cytotoxic factors have non-specific effects on the various functional systems of the organism, even the plant objects;

The organism of patients with myasthenia gravis produce various cytotoxic factors, which influence on different systems of the organism and can lead to the formation of a variety of clinical phenotypes.

The results of changes in the content of autoantibodies, interleukins and expression of various clinical phenotypes of myasthenia forming endogenous cytotoxic factors, can be attributed to the high variability of these parameters in patients' organism.

Thus, cytotoxic factors induce a broad spectrum of different amounts of autoantibodies to various tissues. Autoantibodies have multiple effects. Binding to cellular components of the liver, lungs, heart and other tissues they change functions of these organs, and in addition, because of their enzymatic activity they induce the cytotoxic factors. Afterwards, they induce new synthesis of autoantibodies. We suppose that the formed net of cytotoxic factors drifts into self-sustaining phase, i.e. it is formed the imprint including the specific regulator system of cell activity, in particular, interleukins (Figure 10).

Autoantibodies have multiple effects. Binding to cellular components of the liver, lungs, heart and other tissues, they change function of these organs, and in addition, because of their enzymatic activity they induce the cytotoxic factors. In favor of this, the data may indicate the change of lymphocyte populations and maintain different patterns at patients.

We suppose that each clinical form of myasthenia gravis is characterized by specific features of cytotoxic factors and, as a consequence, by its own pattern of tissue-specific characteristics. On this basis, the choice of treatment strategy should be different for different clinical forms. At the present stage of our enough limited knowledge, about cytotoxic systems and tissue-specific patterns can be used to test $D$. viridis to choose the tactics of treatment and control of the treatment of these patients. Integrated approaches are needed for screening clinical phenotypes of myasthenia gravis, monitoring the effectiveness of treatment and prognosis.

\section{Conflict of interest}

None

\section{References}

Carr AS, Cardwell CR, McCarron PO, McConville J (2010) A systematic review of population based epidemiological studies in Myasthenia Gravis. BMC Neurology 46: 1-9.

Conrad K, Schlosler W, Hiepe F, Fitzler MJ (2011) Autoantibodies in Organ Specific Autoimmune Diseases: A Diagnostic Reference: PABST, Dresden 149-153.

Leite MI, Jacob S, Viegas S, Cossins J, Clover L, et al. (2008) IgG1 antibodies to acetylcholine receptors in 'seronegative' myasthenia gravis. Brain 131: 1940-1952.

Avidan N, Le Panse R, Brrih AS, Miller A (2014a) Genetic basis of myasthenia gravis - a comprehensive review. Journal of Autoimmunity 52: 146-153.

Avidan N, Le Panse R, Harbo HF, Bernasconi P, Poulas K, et al. (2014b) VAV1 and BAFF, via NFkB pathway, are genetic risk factors for Myasthenia Gravis. Annals of Clinical and Translational Neurology 5: 329-339.

Ercolini AM, Miller SD (2009) The role of infections in autoimmune disease. Clin Exp Immunol 155: 1-15.

Tsuda E, Imai T, Hozuki T, Yamauchi R, Saitoh M, et al. (2010) Correlation of bite force with excitation-contraction coupling time of the masseter in myasthenia gravis. Clinical Neurophysiology 121: 1051-1058.

Sanchez JL, Uribe CS, Franco AF, Jimenez ME, Arcos-Burgos OM, et al. (2002) Prevalence of myasthenia gravis in Antioquia, Colombia. Revista de Neurologia 11: 1010-1012.

Antozzi C (2003) Myasthenia gravis and myasthenic syndrome Neurol Sci 4: 260-263.

D. Aarli JA (2001) Titin, thymoma, and myasthenia gravis Arch. Neurol 58: 869-890.

1. Graus YMF, De Baets MH (1993) Myasthenia gravis: An autoimmune response against the acetylcholine receptor. Immunologic research 1: 78-100.

Imai T, Tsuda E, Toyoshima T, Yoshikawa H, Motomura M, et al. (2011) Anti-ryanodine receptor-positive acetylcholine receptornegative myasthenia gravis: evidence of impaired excitationcontraction coupling. Muscle and Nerve 43: 294-295.

Morel E, Garabedian BV, Eymard B, Raimond F, Bustarret FA, et al. (1988) Binding and blocking antibodies to the human acetylcholine receptor: Are they selected in various myasthenia gravis forms? Immunologic research 3: 212-217.

4. Balestra $B$, Moretti $M$, Longhi $R$, Mantegazza R, Clementi F, et al. (2000) Antibodies against neuronal nicotinic receptor subtypes in neurological disorders. J. Neuroimmunol 102: 89-97.

Leite MI, Waters P, Vincent A (2010) Diagnostic use of autoantibodies in myasthenia gravis. Autoimmunity 43: 371-379.

Berrih AS, Cohen KS, Neumann D, Safar D, Eymard B, et al. (1988) Cellular aspects of myasthenia gravis. Immunologic research 3: 189-199. 
17. Anderson MS, Venanzi ES, Klein L, Berzins SP, Turley SJ, et al. (2002) Projection of an immunological self-shadow within the thymus by the aire protein. Science 298: 1395-1401.

Vernino S, Lennon VA (2004) Autoantibody profiles and neurological correlations of thymoma. Clin Cancer Res 10: 7270-7275.

19. Bottini N, Peterson EJ (2014) Tyrosine phosphatase PTPN22: multifunctional regulator of immune signaling, development, and disease. Annual Review of Immunology 32: 83-119.

20. De La Rica R, Stevens MM (2012) Plasmonic ELISA for the ultrasensitive detection of disease biomarkers with the naked eye. Nature Nanotechnology 7: 821.

21. Le Panse R, Cizeron CG, Cuvelier M, Truffault F, Bismuth J, et al. (2008) Regulatory and pathogenic mechanisms in human autoimmune myasthenia gravis. Ann NY Acad Sci 1132: 135-142.

22. Romi F, Gilhus NE, Aarli JA (2006) Myasthenia gravis: disease severity and prognosis. Acta Neurol Scand 183: 24-25.

23. Gratton SM, Herro A, Bermudez-Magner JA, Guy J (2014) Atrophy and fibrosis of extra-ocular muscles in antiacetylcholine receptor antibody myasthenia gravis. Open Journal of Ophthalmology 4: 117-119.

Bofill M, Janossy G, Willcox N, Chilosi M, Trejdosiewicz LK, et al. (1985) Microenvironments in the normal thymus and the thymus in myasthenia gravis. Am J Pathol 119:462-473

Mantegazza R, Bonanno S, Camera G, Antozzi C (2011) Current and emerging therapies for the treatment of myasthenia gravis. Neuropsychiatric Disease and Treatment 7: 151-160.

26. Kataoka H, Furiya Y, Ueno S (2011) Complete Remission of Generalized Myasthenia Gravis by Corticosteroid Treatment Alone without Thymectomy. Case Rep Neurol 3: 239-241.

27. Chuang WY, Ströbel P, Belharazem D, Toyka KV, Nix W, et al. (2009) The PTPN22gain-of-function+1858T (+) genotypes correlate with low IL-2 expression in thymomas and predispose to myasthenia gravis. Genes and Immunity 8: 667-672.

28. Clouston PD, Saper CB, Arbizu T, Johnston I, Lang B, et al. (1992) Cerebellar degeneration, cancer, and the Lambert-Eaton myasthenic syndrome. Neurology 42: 1944-1950.

Scofield RH (2004) Autoantibodies as predictors of disease. The lancet 363: 1544-1546.

30. Compston DAS, Vinsent A, Newsom DJ, Batchelor JR (1980) Clinical pathological HLA antigen and immunological evidence for disease heterogeneity in myasthenia gravis. Brain 103: 579-601.

31. Cull-Candy SG, Miledy R, Trautmann A, Uchitel OB (1980) The release of transmitter at normal, myasthenia gravis and myasthenic syndrome affected human end-plates. J Physiol (Lond.) 299: 621-638.

Klimova EM, Lavinskaya EV, Bozhkov Al, Kordon TI (2010) Estimation of cytotoxicity degree of pathological serum components using cellular test-system. Biotechnologia Acta 6: 85-91.

Gergalova GL, Lehmus OJ, Skok MV (2010) Investigation of the role of nicotinic acetylcholine receptors in the functioning of the mitochondria. Ukraine Biochemical J 4: 61.

Lequin RM (2005) Enzyme immunoassay (EIA)/enzyme-linked immunosorbent assay (ELISA). Clin Chem 51: 2415-2418.
Parcs DR, Lanier L, Herrengerg LA (1986) Flow cytometry and fluorescense activated cell sortins (Facs). Handbook of Experimental Immunology 302-324.

Booth IR, Edwards MD, Black S, Schumann U, Miller S (2007) Mechanosensitive channels in bacteria: signs of closure? Nature Reviews / Microbiology 5: 431-440.

Quintana FJ, Cohen IR (2004) The natural autoantibody repertoire and autoimmune disease. Biomed Pharmacother 58: 276-281.

Gekht BM, Sanadze AG (2003) Myasthenia gravis: diagnosis and treatment. J Neurology 1: 8-12.

Nevinsky GA, Kanyshkova TG, Buneva VN (2000) Natural catalytic antibodies (abzymes) in normalcy and pathology. Biochemistry (Moscow) 65: 1245-1255.

40. Uzawa A, Kawaguchi N, Himuro K, Kanai T, Kuwabara S (2014) Serum cytokine and chemokine profiles in patients with myasthenia gravis. Clin Exp Immunol 2: 232-237.

Castleman B (1966) The pathology of the thymus gland in myasthenia gravis. Ann NY Acad Sci 135: 496-505.

Walker MB (1934) Treatment of myasthenia gravis with physostigmine. Lancet 1: 1200-1201.

Lang B, Newsom DJ, Wray D, Vincent A, Murray N (1981) Autoimmune etiology for myasthenic (Eaton-Lambert) syndrome. Lancet 2: 224-226.

44. Zouvelou V, Zisimopoulou P, Psimenou E, Matsigkou E, Stamboulis E, et al. (2014) AchR-myasthenia gravis switching to double-seropositive several years after the onset. J of Neuroimmunology 267: 111-112.

45. Oda K (1993) Differences in acetylcholine receptor-antibody interactions between extraocular and extremity muscle fibers. Ann NY Acad Sci 681: 238-255.

46. Yamamoto AM, Gajdos P, Eymard B, Tranchant C, Warter JM, et al. (2001) Anti-titin antibodies in myasthenia gravis: tight association with thymoma and heterogeneity of nonthymoma patients. Arch Neurol 6: 885-890.

47. Takamori M (2008) Autoantibodies against TRPC3 and ryanodine receptor in myasthenia gravis. J of Neuroimmunology 200: 142-144.

8. Kaminski HJ, Kusner LL, Wolfe GI, Aban I, Minisman G, et al. (2012) Biomarker development for myasthenia gravis. Ann NY Acad Sci 1275: 101-106.

49. Lutz HU, Binder CJ, Kaveri S (2009) Naturally occurring autoantibodies in homeostasis and disease. Trends Immunol 30: 43-51.

50. Andersen JB, Heldal AT, Engeland A, Gilhus NE (2014) Myasthenia gravis epidemiology in a national cohort; combining multiple disease registries. Acta Neurol Scand Suppl 198: 26-31.

Klimova EM, Drozdova LA, Belozerov IV, Kudrevich AN (2013) Methods of differential diagnostics of various phenotypes of myasthenia on the background of morpho-functional damage of thymus. International journal of immunorehabilitation 1: 44.

Bozhkov Al, Goltvyanskij AV, Rostama SH (2010) Cell aggregates forming in $D$. viridis Teod. culture: a primary reaction to stress induced by high copper ion concentration. Algologia 2: 151-166.

Teodoresco EC (1906) Observations morphologiques et biologiques sur le genre Dunaliella. Rev Gen Bot 18: 353-371. 
54. Masuk NP (1973) Morphology, systematics, ecology, geographical distribution of Dunaliella. Teod Kiev Science pp: 372.
Klimova EM, Lavinskaya EV (2012) Use of the microalgae $D$. viridis Teodor (Chlorophyta) as a cell bioindicator. Algologia 2: 208-218. 